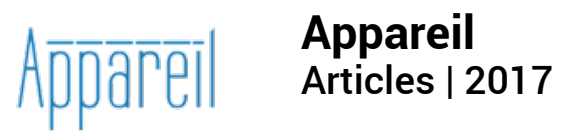

\title{
Lefort/Simondon : Invention démocratique, invention technique
}

Jean-Louis Déotte

\section{(2) OpenEdition}

\section{Journals}

\section{Electronic version}

URL: http://journals.openedition.org/appareil/2373

DOI: 10.4000/appareil.2373

ISSN: 2101-0714

\section{Publisher}

MSH Paris Nord

\section{Electronic reference}

Jean-Louis Déotte, «Lefort/Simondon : Invention démocratique, invention technique », Appareil [Online], Articles, Online since 12 May 2017, connection on 30 July 2020. URL : http:// journals.openedition.org/appareil/2373; DOI : https://doi.org/10.4000/appareil.2373

This text was automatically generated on 30 July 2020 .

\section{(c) (i) (9)}

Appareil est mis à disposition selon les termes de la Licence Creative Commons Attribution - Pas d'Utilisation Commerciale - Pas de Modification 4.0 International. 


\title{
Lefort/Simondon : Invention démocratique, invention technique
}

\author{
Jean-Louis Déotte
}

1 Je distinguerai à l'intérieur d'une histoire de la technique, des régimes symboliques de la technique en fonction des trois grands modes de légitimation des discours que sont la narration, la révélation, la délibération objectivante. Pour établir ces distinctions, je m'appuierai aussi bien sur Lefort: Les Formes de l'histoire, que sur Lyotard: Le Différend qui le prolonge.

2 Le chaman opère en récitant des mythes que tous les membres de sa communauté connaissent, le prêtre chrétien soigne les âmes en se référant aux Ecritures données à voir sous la forme d'images ou d'icônes, le chirurgien applique une technique acquise à l'Université, dont il peut essentiellement rendre raison. Ces normes de légitimation sont indérivables les unes des autres, elles ne dépendent pas d'un état du savoir ou du pouvoir, au contraire, elles introduisent à chaque fois, comme le rappelle Lefort ${ }^{1}$, un rapport déterminé entre le savoir, le pouvoir et la loi.

3 Cette distinction concerne aussi les supports où s'inscrivent les traces: un plan de projection (comme l'est le tableau comme fenêtre inventé par la Renaissance albertienne) n'est pas le plan de l'écran numérique de nos ordinateurs. De même que le premier se distinguait du plan d'inscription des signes de la peinture-enluminure médiévale ${ }^{2}$. En rapport avec les modes de légitimité, il faut donc distinguer des modes de la proto-écriture ou proto-géométrie : à la narration "sauvage » correspond une écriture sur les corps qui n'est pas lisible au sens strict. Contrairement à ce qu'affirmait Clastres $^{3}$, les traces sur les corps (cicatrices rituelles, tatouages, circoncision, excision, etc.) ne constituent pas un texte donné à lire. Dans les « sociétés contre l'État " qu'il observe, le rapport entre l'individu et la loi est alors sans médiation, en dehors du rituel d'écriture qui est un véritable appareil : l'individu est strictement pour la loi. Dans le cas des sociétés du Livre, soumises à la révélation et à sa médiation, le message génère son médium. Le livre n'est pas moins sacré que le texte qui peut consister en un ordre géométrique élémentaire comme dans le cas des muquarnas de l'architecture musulmane, que les Occidentaux prirent pour de la décoration. La révolution projective 
surgissant en Italie à la Renaissance, est, elle, indissociable du mode délibératif : on ne peut représenter sur un plan transparent que ce dont on est dissocié, définitivement. L'appareil perspectif devient alors la condition de possibilité de l'objectivation du monde, laquelle entrâne la nécessité d'une discussion sans fin sur les buts et moyens de la Cité politique. C'est l'horizon des textes de Machiavel, le Prince comme les Discours.

Entre les proto-écritures que nous avons distinguées, pas plus qu'entre les normes de légitimation ou ordres symboliques, il n'est concevable d'imaginer des continuités. Ce qui entraîne Simondon, cet essentiel penseur de la technique et de ses phases, mais aussi des époques de la culture, à de nombreux paradoxes, sur lesquels je reviendrai. Par exemple, comment concevoir que le monde "magique $»^{4}$, celui de la stricte hétéronomie (les Grands Ancêtres), où les hommes disent agir toujours de même depuis la nuit des temps parce que c'est Bien, comment aurait-il pu être bouleversé par un événement d'une importance suffisante pour le faire évoluer vers tout autre chose que son ordre symbolique? C'est une question que retrouve Clastres quand il doit aborder la question du pouvoir effectif. Simondon en arrive alors à affirmer qu'il y a des régimes de l'hominisation, caractérisés par une articulation métastable entre les figures et les puissances de fond, développant des techniques comme la chasse collective, sans qu'ils donnent lieu à des objets techniques. C'est accepter que ces sociétés holistes ne puissent être bouleversées par une invention technique, car comme nous l'avons dit, s'il s'en présente une du fait d'envahisseurs par exemple, elle est immédiatement attribuée aux Grands Ancêtres qui ont légué par avance à la société son ordre, qui, par définition, est Bien. L'invention technique est alors renvoyée en avant, du côté d'un temps qui n'est pas humain. Si un certain ordre symbolique est capable de ne pas rencontrer de problèmes donnant lieu à une invention technique, ou alors d'une manière qui ne laissera pas de traces, c'est que le symbolique, tout en étant proprement appareillé (par la narration par exemple), peut ou non promouvoir l'invention d'objets techniques.

5 Ainsi, est-il évident qu'une simple description qui s'en tient à l'état des techniques ou des échanges, ne peut rendre compte de ce qui sera un véritable avènement : la rupture de l'ordre symbolique qui donnait tous les repères de la certitude, par le surgissement d'un nouvel ordre dont on ne peut rendre compte par l'excroissance de telle ou telle logique. Paul Veyne ${ }^{5}$, dans un livre récent sur Constantin et l'adoption du christianisme à Rome la païenne, a montré qu'aucune raison, "idéologique», politique, sociale, dynastique, etc., ne pouvait en rendre compte. Il se trouva simplement que l'Empereur, pour des raisons définitivement impénétrables, que les Chrétiens appellent la grâce, a fait un choix privé, qui n'a finalement bouleversé progressivement l'ensemble de l'Empire que du fait de sa position éminente. Le culte du premier des Romains est devenu peu à peu celui de l'ensemble impérial, pour des raisons finalement sociologiques : l'avènement du nouvel ordre symbolique a peu à peu fait tache d'huile en ne répondant à aucune logique extrinsèque. Ce ne sont pas des raisons éthiques, comme l'exécration des combats du cirque ou de l'exploitation des esclaves, qui auraient imposé le nouvel ordre, parce que les Romains christianisés continuèrent d'aller au cirque et d'avoir des esclaves. Il s'en est d'ailleurs fallu de peu, qu'à de nombreuses reprises, ce nouvel ordre symbolique ne soit abandonné et que Rome réintègre massivement le paganisme. Nous ne voulons pas faire ici seulement l'éloge de la contingence dans l'histoire, ni finalement celle de l'événement, mais rappeler qu'il y a des discontinuités radicales, dont ne peut rendre compte l'ordre symbolique précédent, parce que les normes de légitimation sont indérivables. Les raisons 
invoquées en général pour expliquer l'adoption du Christianisme sont toujours déjà chrétiennes. Entre le paganisme et la Révélation, il ne peut y avoir de passage parce qu'il y a discontinuité radicale, symbolique. Le fait du paganisme, qui consistait pour le Romain à entretenir de bons rapports avec telle ou telle divinité de son choix, avant de l'abandonner pour une plus efficace, et donc à lui faire des offrandes puis cesser, ou, s'il était Empereur, à lui édifier un temple, ne peut permettre de comprendre comment tel ou tel Romain a pris la décision de s'incarner dans le corps du Christ au moment où ce dernier s'incorporait en lui au moment de l'Eucharistie. Cela étant Veyne, trop sociologue, minimise certainement l'attrait pour la nouvelle forme de la communauté, fondée non plus sur l'autorité du pater familias, mais sur l'incorporation. L'Empire ne proposait que la Totalité, le Christ : l'Un.

6 Mais alors, pour l'essentiel, le régime symbolique de la Révélation, privilégia les techniques réduites à des sémio-techniques : commentaires du texte sacré, édification d'architectures de destination (églises, mosquées), développement d'une peinture devenant la face visible du texte lisible, etc. Ce qui n'est pas contraire avec une rationalisation de la foi (le thomisme). D'où l'importance de la formation dans les monastères, dans les écoles cathédrales et finalement à l'Université, en particulier à Paris.

7 Ainsi l'état des techniques resta, grosso modo, celui des Romains pendant plus d'un millénaire. Jusqu'à l'irruption de la proto-écriture projective (Brunelleschi, Florence, $\mathrm{xv}^{\mathrm{e}}$ siècle, puis Léonard da Vinci, l'ingénieur) qui est fondamentalement une révolution de la surface d'inscription, c'est-à-dire du rapport entretenu par les signes (les traces) avec la loi.

8 Ce qu'on appelle "époque de la technique ", serait donc ce moment où les techniques, relevant du principe de raison, sans être pour autant déjà des techno sciences, sont l'objet d'une délibération constante à l'intérieur d'un groupe et donc aussi bien à l'«intérieur" de l'esprit d'un individu attaché à la résolution de problèmes: le technicien comme inventeur. Je propose donc de corréler l'invention des premiers « objets techniques » au sens de Simondon (bien plus que des outils, des instruments ou des appareils au sens restreint comme le microscope ou la lunette astronomique) avec les différents régimes de légitimation des modes d'enchaînement des discours. Proposons un exemple: le moteur à combustion interne, révolutionnaire au sens d'indérivé et ouvrant une lignée phylogénétique, avec bien des enrichissements ultérieurs. En fait, quand Simondon ${ }^{6}$ analyse l'objet technique en termes de concrétisation, de synergie, de convergence des fonctionnalités, de création d'un milieu naturel «associé ", transformé par l'incorporation de certaines structures naturelles, on voit bien qu'un objet technique accompli prépare le terrain pour la science qui, elle seule, comme mode de légitimation, actualise la quintessence du principe de raison. Car on ne peut nécessairement rendre compte tout de suite scientifiquement d'une invention technique.

9 Simondon a révolutionné notre compréhension du développement technique en refusant l'horizon qui était le sien jusqu'alors, celui de l'usage (le rapport aristotélicien moyens/fins) pour s'intéresser au fonctionnement des objets techniques. On ne connaît un tel objet que si l'on est capable de le démonter/remonter. Avec lui, la technique est réintroduite dans la culture et finalement dans la philosophie qui la réfléchit.

10 Revenons sur le processus de concrétisation comme auto-corrélation: le progrès technique va consister à réduire l'abstraction des objets techniques plus primitifs en 
réduisant la divergence des éléments techniques. En effet, un élément de cet objet ne se réduit pas à accomplir une fonction, sa perfection consiste à accomplir plusieurs fonctions (cf. l'ailette d'un cylindre de piston qui renforce physiquement le piston et élimine en même temps davantage de chaleur). Mieux, la convergence des fonctionnalités détermine une synergie de tous, une résonance telle, qu'un élément qui ne remplirait qu'une fonction serait inessentiel, «abstrait». Le devenir des objets techniques semble répondre à l'idéal vitaliste et organiciste, celui de l'autonomie des êtres naturels. Mais c'est une fausse utopie, celle de l'androïde par exemple, que récuse Simondon. Nous insisterons donc sur cette sorte de débat constant entre elles des pièces d'un moteur, avec comme horizon inatteignable, l'autonomie relative de l'être vivant quant au milieu (biotope), et comme facteur régulier, une sorte de désaccord entre les organes et les éléments pouvant aller jusqu'à la "sursaturation » rendant nécessaire le passage à autre chose, à un autre rapport entre les éléments, entre l'objet technique et son milieu. À la lecture de Simondon, on comprend qu'un déséquilibre interne fait problème et que la résolution consiste dans une meilleure connexion, dans une meilleure relation entre les termes qui constituent un réseau. Que la crise et sa résolution soient les clefs de l'évolution d'un objet technique, puis d'un ensemble technique (comme l'est un réseau ferroviaire ou le carreau d'une mine organisé autour du puits de mine), voilà qui n'était réalisable et pensable qu'à l'âge démocratique, inauguré selon Lefort par Machiavel'7.

La thèse serait la suivante: c'est l'âge démocratique, qui allie représentation et capitalisme, qui a libéré la logique technique de la contrainte des corporations médiévales, lesquelles vivaient suivant les repères hiérarchiques et traditionnels d'un régime théologico-politique. Il aura fallu, comme le répète souvent Lefort, une perte des repères de la certitude et une désincorporation des pôles du pouvoir, du savoir et de la loi, pour que toutes les voies possibles de l'invention technique soient explorées et qu'à partir de ces inventions, des connexions inouies entre les objets techniques s'imposent, générant de nouveaux ensembles techniques.

Quand Heidegger critique la Modernité, il s'en prend d'une manière cohérente à la technique et à la démocratie. S'attaquer à l'une, c'est s'attaquer à l'autre, parce que l'une et l'autre font l'épreuve de l'incertitude. Et donc de la crise. Sans légitimation de la crise interne, il n'y a pas de raison qu'une société ou un objet technique évoluent. Sans débat, sans incertitude fondamentale, il n'y a pas d'inventions possibles puisque sinon les solutions sont toujours données d'avance et totalement contraignantes.

Il est évident que les techniques, soumises au régime démocratique-capitaliste ne peuvent aller toujours jusqu'au bout de leur logique, en particulier si elles ne semblent pas déboucher sur une application rapide. Malgré l'autonomie de la logique technicienne, les techniques ne sont pas absolument libérées du libéralisme économique ou des contraintes politiques du totalitarisme. Mais ce qui a été génétiquement promu n'est pas oublié malgré le corset d'une culture historique. Et inversement, des événements peuvent surgir en leur sein déstabilisant l'ensemble du marché et de la production comme on le voit avec l'introduction d'internet et de la norme www. Personne n'avait prévu qu'une norme élaborée dans le cadre d'un centre de recherche en physique fondamentale, le CERN à Genève, allait bouleverser les communications mondiales et le marché au sens le plus large, en provoquant la révolution la plus considérable connue par l'humanité. 
Il y avait chez les philosophes issus de Socialisme ou Barbarie (outre Lyotard et Lefort, Damisch et Morin, etc.) la possiblité d'une synthèse supérieure que Simondon aurait pu appeler "allagmatique " (science des opérations génétiques, science transversale et unifiante). En effet, et on s'étonne toujours de ce manque de convergence interne, entre Lefort, penseur de l'invention démocratique, Lyotard des époques de la surface d'inscription puis du différend, Damisch de l'origine de la perspective, Morin du double comme support de la projection. Tous visent la même césure historique, la rupture avec l'ordre théologico-politique et l'irruption inouie de l'écriture projective, sans se rencontrer. Sans imaginer le sol époqual commun, par exemple entre l'insurrection des Ciompi à Florence au XIV ${ }^{\mathrm{e}}$ siècle, la lecture perspectiviste que développa Machiavel de Tite Live, la tradition picturale chrétienne de Sienne de laquelle Brunelleschi s'affranchit en faisant construire la double coupole de Sainte Marie de la Fleur à Florence et en donnant à voir les conditions de l'appareil perspectif. La division académique impose toujours qu'un philosophe du politique ne peut s'intéresser à l'esthétique et inversement. Qu'est-ce qui leur a manqué ? Un constat et une médiation, ceux que postule Benjamin dès l'ouverture de L'œuvre d'art à l'époque de sa reproductibilité technique: il y a des époques de la sensibilité humaine, car celle-ci est appareillée techniquement. Pour le $\mathrm{xx}^{\mathrm{e}}$ siècle, cet appareil projectif, c'est le cinéma. Pour le $\mathrm{XIX}^{\mathrm{e}}$ siècle, la photographie, le musée et le monument patrimonial, pour les siècles antérieurs, la camera obscura, la perspective, la lanterne magique, etc. ${ }^{8}$. Un appareil configure les apparences selon des proto-écritures; ici la proto-écriture projective, condition de possibilité de la perspective est à la fois requise pour la révolution renaissante des arts et pour la science galiléenne, c'est-à-dire pour toute la science moderne. C'est une invention absolue sur le plan phylogénétique. Si l'on ne peut ignorer ce qu'il en a été pour les arts (peinture, sculpture, architecture, théâtre, etc.) qui sont tous passés sous la coupe du disegno et pour la science où la quantification a évincé la qualité, on ne s'est pas interrogé sur la nouvelle définition de la politique. En abandonnant l'incarnation théologico-politique, comme Hobbes l'a montré dans le Léviathan ${ }^{9}$, c'est l'ordre de la représentation qui s'est imposé. Ce que Lefort appelait idéologie ou Benjamin fantasmagorie, puisque notre espace commun en tant qu'espèce, c'est déjà l'image et le rêve plus que le langage.

Alors qu'ils en avaient les moyens (pensons aux travaux de Morin sur le cinéma, à ceux de Lyotard sur la psychanalyse, etc.), nos philosophes issus de SOB, trop marqués par la phénoménologie, ont fait l'impasse sur les époques du politique qui sont autant d'époque de l'appareillage de l'événement. Je n'ignore pas l'exposition exemplaire de Lyotard au Centre G. Pompidou en 1985 : Les immatériaux. À l'aube d'une nouvelle protoécriture, le numérique, sur la base d'un autre paradigme scientifique, la théorie de la communication élaborée par Shannon, Lyotard a renouvelé radicalement sa théorie de la surface d'inscription élaborée dans Discours, Figure. Après ce coup de force, il ne reste pas grand-chose des grands récits, de l'émancipation, du travail, de la volonté, de la nature, de la matière, sauf les algorithmes. Et puis le souffle lyotardien est retombé et le grand texte sur le post-colonialisme, Le Différend, fait l'éloge du pur événement qu'est la phrase qui ignore tout support.

Actuellement, parce que l'écriture projective était grosse de l'écriture numérique, comme c'était déjà évident chez Alberti avec son plan de Rome, la continuité semble l'emporter sur la discontinuité : en apparence nos appareils numériques ne sont que des perfectionnements des appareils analogiques. 

arts, tous les appareils de la modernité, qui étaient projectifs, sont très rapidement intégrés par le numérique. Mais ce qu'ils produisent comme images et comme sons est tout autre parce que le rapport à la temporalité qu'ils inventent est lui aussi tout autre. On ne pourrait pas à partir d'une photo numérique développer l'analyse barthésienne du ça a été parce que l'image numérique n'entretient plus de rapport physique à l'existant. Et c'est bien là encore, la temporalité qui est en jeu. Comme on le sait, cette révolution technique de la communication n'a pas été non plus sans effets sur la discussion politique. Il est évident qu'il faut reprendre à nouveaux frais l'analyse de la démocratie comme du totalitarisme à partir du moment où les savoirs, les savoir-faire, le langage lui-même sont externalisés. d'innervation du corps collectif de l'humanité par les techniques imageantes, ce qui inclut la littérature. C'est dire que le psycho-social et les techniques, époque après époque, marchent de pair. C'est d'autant plus vrai pour le «collectif réel» que Simondon distinguait de la communauté, par exemple d'un groupe d'ouvriers où règnent les relations inter-individuelles, celles qu'étudie le sociologue du travail. Un collectif réel ou transindividuel est indissociable d'une technique. Pour que cette relation, dite transindividuelle, s'établisse il faut qu'une technique imageante permette à chacun de s'individuer en entraînant ainsi une individuation de son groupe. On en a un contre-exemple dans le livre que Denis Skopin a consacré à la censure des photographies de groupe en URSS en 1937 dans la région de Nijni-Novgorod. Il ne s'agissait pas tant pour la répression de faire disparaitre à des fins d'archivage telle ou telle figure marquante et on connaît tous ces photos du Comité Central qui étaient élaguées ${ }^{10} d$ 'année en année, jusqu'à ce que seul le leader subsistât, seule figure de proue de la Révolution.

19 La tâche du NKVD était autre : un groupe étant constitué par une photo, et n'ayant d'existence que par elle, il s'agissait sous la pression la plus extrême de transformer tel ou tel membre en un liquidateur de la photo du parasite. C'était à lui de biffer, de caviarder, de recouvrir d'encre le visage de l'ami, du camarade, du leader. La destruction $\mathrm{du}$ groupe passait par ce qui l'avait constitué comme tel. La désindividuation du groupe et donc de chacun de ses membres était accomplie quand tous retombaient dans le pré-individuel.

À partir de cet exemple, qui remet en cause l'interprétation lefortienne du totalitarisme, trop inféodée à une phénoménologie de l'incarnation, on comprend que les logiques du politique et de la technique ne peuvent être dissociées car les groupes humains sont intrinsèquement technologiques.

\section{BIBLIOGRAPHY}

Clastres, P., La société contre l'État, Paris, Minuit, 1974. 
Crignon, P., De l'incarnation à la représentation - L'ontologie politique de Thomas Hobbes. Paris, Garnier, 2012.

Kittler, S., Les Médias optiques. Cours de Berlin de 1999. Ouvr. coll. édité par Audrey Rieber, Paris, L'Harmattan, trad. fr. 2015.

Lefort, Cl., Les Formes de l'histoire. Essais d'anthropologie politique. Paris, Gallimard. 1978.

Lefort, Cl., Machiavel, le travail de l'œuvre, Paris, Gallimard, 1972.

Lyotard, J.F., Discours, Figure, Paris, Klincksiek,1972.

Simondon, G., Le mode d'existence des objets techniques. Paris, Aubier, 1958.

Skopin, D., La photographie de groupe et la politique de disparition dans la Russie de Staline. Paris, L'Harmattan, 2015.

Veyne, P., Quand notre monde est devenu chrétien, Paris, Albin Michel, 2007.

\section{NOTES}

1. Cl. Lefort : Les Formes de l'histoire. Essais d'anthropologie politique. Paris. 1978.

2. J.F. Lyotard : Discours, Figure, Paris, 1972..

3. P. Clastres : La société contre l'Etat, Paris, 1974.

4. G. Simonon : Le mode d'existence des objets techniques. Paris, 1958.

5. P.Veyne: Quand notre monde est devenu chrétien, Paris, 2007.

6. Simondon: Du mode d'existence des objets techniques (MEOT).

7. Cl. Lefort : Machiavel, le travail de l'œuvre. Paris, 1972.

8. S. Kittler: Les Médias optique. Cours de Berlin de 1999. Ouvr. coll. édité par Audrey Rieber, Paris, trad. fr. 2015.

9. P. Crignon : De l'incarnation à la représentation - L'ontologie politique de Thomas Hobbes. Paris, 2012.

10. D. Skopin : La photographie de groupe et la politique de disparition dans la Russie de Staline. Paris, 2015.

\section{ABSTRACTS}

L'évolutionnisme radical de Simondon, en particulier sa théorie de la culture technique, fait l'impasse sur les événements de l'histoire des hommes qui sont de l'ordre de l'instauration de nouveaux ordres symboliques ou formes de l'histoire (Lefort). Inversement la philosophie politique de Lefort ne se préoccupe pas des appareils qui ont conditionné la sensibilité collective et donc l'accueil de l'événement politique. Cet article se propose donc de cerner un milieu commun à la politique, à la technique comme à l'esthétique. 
INDEX

Mots-clés: invention démocratique, invention technique, formes de l'histoire, perte démocratique des repères, Lyotard

\section{AUTHOR}

\section{JEAN-LOUIS DÉOTTE}

Professeur émérite de philosophie, Université Paris VIII-MSH Paris Nord. 